ORIGINAL ARTICLE

\title{
Breast cancer recurrence: follow up after treatment for primary breast cancer
}

\section{N Hiramanek}

Background: Breast cancer will affect one in 12 Australian women in their lifetime. After potentially curative treatment, it is usual for patients to be followed up for many years. However, controversies surround follow up, and its value is uncertain.

Aim: This study reviews the evidence and describes the role of routine follow up in the management of breast cancer in a tertiary hospital. By establishing how recurrence of breast cancer presents and identifying the proportion of patients with recurrence who were diagnosed as a consequence of regular follow up, the value of this system in detecting recurrence can be indirectly assessed.

Design: A retrospective review was undertaken of all patients attending the radiotherapy outpatient clinic for treatment of invasive breast cancer in 1997. Hospital records were reviewed to ascertain patient and

Correspondence to:

Dr N Hiramanek

University of Sydney, 37a

Booth Street, Balmain,

Sydney, NSW 2041,

Australia; navazh@

med.usyd.edu.au

Submitted 5 June 2003

Accepted

8 September 2003 tumour characteristics, treatment received, site of recurrence and its mode of detection, whether patients were symptomatic at the time of recurrence, and whether they presented at a scheduled appointment.

Results: Out of 286 patients who presented to the radiotherapy outpatient clinic, 220 were entered into the study. Recurrence was recorded in 42 patients (a complete dataset was available for 38 of these patients). In total, 74\% (31/42) of recurrences were symptomatic, and 76\% (32/42) presented at unscheduled appointments, the majority (17/32) of which were initiated by the patient. Only seven patients had asymptomatic recurrences.

Conclusion: Based on current evidence, long term routine hospital follow up after treatment for breast cancer appears to be inefficient in detecting recurrence.
B reast cancer is an important public health problem, affecting one in 12 Australian women in their lifetime. ${ }^{1}$ After potentially curative treatment for breast cancer it is common clinical practice for patients to be followed up for many years. However, controversies surround follow up, and its value is uncertain.

International figures suggest that about $30 \%$ of women will develop recurrence after treatment for primary breast cancer, figures for early stage disease being lower. ${ }^{23}$ The available data confirm that Australian recurrence rates are similar to the international average. ${ }^{4-6}$ Today, the combined effects of screening, early detection, and advances in treatment have resulted in increasing numbers of patients diagnosed with breast cancer in follow up clinics. At the same time, there is increasing pressure on resources from new referrals and urgent cases.

Follow up should include providing physical and psychosocial rehabilitation, monitoring treatment effectiveness and short term and long term toxicity, detecting recurrences and new cancers, and collecting data for research. Follow up schedules vary internationally. Guidelines issued by the American Society of Clinical Oncology in 1999 recommend monthly breast self examination, annual mammography of the preserved and contralateral breasts, and a history and physical examination every three to six months for three years, then every six to 12 months for two years, then annually. ${ }^{7}$ Guidelines issued by the National Health and Medical Research Council of Australia recommend annual mammography of the preserved and contralateral breasts, and a history and physical examination every three months for two years, then every six months for three years, then annually. ${ }^{1}$ These recommendations are resource intensive.

Evidence that intensive follow up is of little value has come from randomised controlled trials and a Cochrane review. The Cochrane review of follow up strategies for women treated for early breast cancer (based on four randomised controlled trials) concluded that follow up programmes based on regular physical examinations and yearly mammography alone appear to be as effective as more intensive approaches based on regular laboratory and instrumental tests in terms of timeliness of recurrence detection, overall survival, and quality of life..$^{8-11}$

Routine follow up protocols in use today are based on two hypotheses: (i) that most recurrences are detected at an earlier stage through follow up; and (ii) that the earlier treatment of recurrences offers a better chance of cure, longer survival, or improvement in quality of life. However, current data suggest that neither of these hypotheses is correct and that postoperative follow up of patients with breast cancer is costly and time consuming and does not significantly extend survival. ${ }^{12-14}$

Despite considerable effort to detect recurrent disease early, the evidence suggests that only a minority of recurrences are detected at an asymptomatic stage. ${ }^{15} 16$ It has also been found that most patients with recurrence have signs or symptoms as the first indicator of recurrence and that history and physical examination generally provide the first clues to recurrence. ${ }^{17}{ }^{18}$

Furthermore, most recurrences present at unscheduled appointments and not as a consequence of routine follow up appointments. ${ }^{15} 1619$

When analysing the presentation of recurrent breast cancer, a distinction has been made between locoregional and metastatic disease. Metastatic disease is incurable, and it has been demonstrated that no survival advantage is gained from diagnosing it at an asymptomatic stage by means of routine investigations. ${ }^{17}$

Whether early detection of locoregional recurrence leads to a survival benefit, however, is still controversial, with the current evidence suggesting that early detection and 
treatment of asymptomatic locoregional recurrence has no benefit to overall survival compared with treatment of symptomatic recurrence. ${ }^{15}{ }^{19-22}$

Furthermore, the assumption that early detection of asymptomatic locoregional recurrence is associated with a better chance of controlling disease symptoms and thereby improving quality of life is also unproven. It should be noted, however, that this situation is different after breast conservation surgery, where salvage mastectomy is a well recognised treatment. ${ }^{23}$

Since breast cancer recurrence usually presents to an interval clinic, and most cases that are confirmed following a routine review are already symptomatic, the efficacy of routine follow up in hospital outpatient clinics is highly questionable..$^{13} 151719$

There is no evidence as to what constitutes optimal follow up, and identification of an optimised follow up strategy will require long term prospective clinical trials. In the meantime, I sought to determine the value of routine hospital follow up indirectly by identifying the proportion of women with recurrent breast cancer who were asymptomatic and diagnosed directly as a result of routine outpatient attendance.

\section{PATIENTS AND METHODS}

I conducted a retrospective cross sectional study of patients who attended a tertiary hospital radiotherapy outpatient clinic in 1997 with a diagnosis of invasive breast cancer. Ethical approval to conduct the study was obtained from the hospital ethics committee, and the study was conducted with due attention to patient confidentiality.
Out of a total of 286 patients who presented, 220 patients were entered into the study. The remaining 66 patients were excluded because they had metastasis at the time of diagnosis. Data on 220 patients were examined to determine the pattern of recurrence over a five year follow up period. Full five year follow up on 65 patients was available from their hospital outpatient records; for the remainder, the patient's surgeon or oncologist was contacted to achieve a five year follow up.

The management and frequency of follow up visits were determined by the specialist, protocol at the time, and clinical indications, but visits generally occurred at intervals of six months for two years and then annually to five years. Annual mammography was performed; no other investigations were routinely undertaken.

Recurrences were classified as locoregional if they occurred in the ipsilateral breast or in the axillary or supraclavicular lymph nodes, and as distant metastasis if they occurred at any other site. Tumours in the contralateral breast were recorded separately. These may have been new primary cancers or metastases from the previous primaries. Recurrences were classified as symptomatic if the patient presented with a relevant complaint. Clinic visits were classified as scheduled if they corresponded to a routine follow up appointment, irrespective of whether the patient had symptoms. If patients were referred back to the clinic between routine appointments then these visits were classified as unscheduled. No attempt was made to quantify counselling or support requested or delivered at clinic visits.

Analyses identified the site of recurrence, the recurrence rate, whether the recurrence was symptomatic or

Table 1 Patient and tumour demographics and treatment received

\begin{tabular}{|c|c|c|}
\hline \multirow[b]{2}{*}{ Category } & \multirow{2}{*}{$\begin{array}{l}\text { All patients }(n=220) \\
\text { No }(\%)\end{array}$} & \multirow{2}{*}{$\begin{array}{l}\text { Patients with recurrence }(n=42) \\
\text { No }(\%)\end{array}$} \\
\hline & & \\
\hline \multicolumn{3}{|l|}{ Age } \\
\hline$<50$ years & $90(41)$ & $10(24)$ \\
\hline$\geqslant 50$ years & $130(59)$ & $32(76)$ \\
\hline \multicolumn{3}{|l|}{ Histology } \\
\hline Infiltrating ductal including no special type & $164(75)$ & $26(62)$ \\
\hline Infiltrating lobular & $23(10)$ & $10(24)$ \\
\hline Tubular or cribriform & $10(5)$ & $0(0)$ \\
\hline Mucinous or colloid adenocarcinoma & $5(2)$ & $1(2)$ \\
\hline Other carcinoma of mixed type & 5 (2) & $1(2)$ \\
\hline Papillary & $0(0)$ & $0(0)$ \\
\hline Medullary & $0(0)$ & $0(0)$ \\
\hline Unknown or not in file & $13(6)$ & $4(10)$ \\
\hline \multicolumn{3}{|l|}{ Grade } \\
\hline Well differentiated, low grade, G1 & $47(21)$ & $2(5)$ \\
\hline Intermediate, medium grade, G2 & $74(34)$ & $13(31)$ \\
\hline Poorly differentiated, high grade, G3 & $74(34)$ & $19(45)$ \\
\hline Unknown or not in file & $25(11)$ & $8(19)$ \\
\hline \multicolumn{3}{|l|}{ Tumour size } \\
\hline$<2 \mathrm{~cm}$ & $119(54)$ & $15(36)$ \\
\hline$\geqslant 2 \mathrm{~cm}$ but $<5 \mathrm{~cm}$ & $75(34)$ & $19(45)$ \\
\hline At least $5 \mathrm{~cm}$ & $11(5)$ & $5(12)$ \\
\hline Unknown or not in file & $15(7)$ & $3(7)$ \\
\hline \multicolumn{3}{|l|}{ Nodes } \\
\hline Negative & $118(54)$ & $15(36)$ \\
\hline $1-3$ positive nodes & $37(17)$ & $8(19)$ \\
\hline$\geqslant 4$ positive nodes & $40(18)$ & $15(36)$ \\
\hline Nodes not examined & $25(11)$ & $3(7)$ \\
\hline Unknown or not in file & $0(0)$ & $1(2)$ \\
\hline \multicolumn{3}{|l|}{ Oestrogen receptor } \\
\hline Positive & $145(66)$ & $21(50)$ \\
\hline Negative & $34(15)$ & $9(21)$ \\
\hline Unknown or not in file & 41 (19) & $12(29)$ \\
\hline \multicolumn{3}{|l|}{ Treatment } \\
\hline Surgery & $214(97)$ & $42(100)$ \\
\hline Radiotherapy & $188(85)$ & $35(83)$ \\
\hline Hormonal & $125(57)$ & $25(60)$ \\
\hline Chemotherapy & $75(34)$ & $28(67)$ \\
\hline
\end{tabular}


Table 2 Site of first recurrence

\begin{tabular}{ll}
\hline Site of first recurrence & No $(\%)$ of patients $(\mathbf{n}=\mathbf{2 2 0})$ \\
\hline Metastatic & $25(11)$ \\
Locoregional (same breast or ipsilateral & $9(4)$ \\
regional nodes) & $4(2)$ \\
Contralateral breast & $4(2)$ \\
Unknown or not in file & \\
\hline
\end{tabular}

asymptomatic, the first indicator of recurrence, whether it was identified at a scheduled or an unscheduled visit, the referral pattern for unscheduled visits, and what proportion of recurrences were detected as a result of follow up in the clinic (that is, diagnosed by a clinic doctor at a scheduled visit in an asymptomatic patient).

The patient and tumour characteristics at diagnosis and the treatment given are shown in table 1 . The characteristics of patients with recurrence are also shown. However, because of the small numbers and because this was not an aim of the study, I will not draw any further conclusions about prognostic indicators of recurrence.

\section{RESULTS}

Recurrence was recorded in 42 patients (19\%) (a complete dataset was available for 38 of these patients), with a disease free interval ranging from 10 months to five years. Patients with recurrence were categorised according to the site of recurrence (table 2). Overall, 25 patients developed metastatic disease; the most common site of metastasis was bone. Table 3 details the recurrence rate at intervals of six months. Most recurrences occurred in the second or third year after diagnosis. The recurrence rate varied between $0.5 \%$ and $7.9 \%$ with no clearly consistent pattern.

The most common first indicator of recurrence (table 4) was patient symptoms or the finding of an abnormality on self examination (31/42), with the majority of patients presenting with pain. Specialist examination picked up two cases, and a routine mammogram was the first indicator of recurrence in five cases.

Table 5 categorises recurrence by visit type, presence or absence of symptoms, and site of recurrence. Of those patients whose disease recurred, $74 \%$ were symptomatic, and $76 \%$ of recurrences presented at unscheduled appointments. Looking at the referral pattern for unscheduled visits (table 6 ), it is seen that $53 \%$ were initiated by the patient, and $31 \%$ of patients were referred by their general practitioners. Only seven patients had asymptomatic recurrences; five of these were detected by mammography, and only two were detected by physical examination in the outpatient clinic (representing 1\% of the 220 patients followed up).

\section{DISCUSSION}

In this study recurrence was recorded in 42 patients (19\%). These data, like those of others, ${ }^{17}{ }^{18}$ show that most recurrences $(74 \%)$ are symptomatic and that signs and symptoms are the most frequent indicator of recurrence.

Boekhorst et al reported that only $37 \%$ of recurrences were detected while they were asymptomatic, ${ }^{15}$ and Loong et al found that only 24 out of 115 patients with recurrence were diagnosed before they became symptomatic. ${ }^{16}$ In a study by Pandya et al the first indicator of recurrence was symptoms in $36 \%$, patient self examination in $18.3 \%$, physical examination by the physician in $19.4 \%$, abnormal blood chemistries in $12 \%$, bone scans in $8 \%$, chest radiographs in $5.1 \%$, and mammograms in $1.1 \%{ }^{18}$

In this study $76 \%$ of recurrences were diagnosed at unscheduled appointments, which were often initiated by the patient. Similar results were obtained in other studies: after a median follow up period of 76 months, Loong et al reported that $55 \%$ of local recurrences and $70 \%$ of metastatic recurrences were detected by interim referral, ${ }^{16}$ and Churn and Kelly found that $64 \%$ of local recurrences were detected at interim referral. ${ }^{19}$

This study found that only two recurrences were detected directly as a result of routine follow up, representing $1 \%$ of the 220 patients being followed up. This pilot study was limited by assessing follow up in only one outpatient clinic (hospital based follow up usually occurs over a few different clinics) and by small numbers, incomplete data collection, low statistical power, and retrospective data collection. However, my findings are in accordance with those of larger studies. ${ }^{16} 1719$

Morris et al discovered that only $1 \%$ of routine visits contribute to the detection of recurrence following potentially curative treatment of breast cancer, compared with $27 \%$ of interval visits. ${ }^{24}$ Holli and Hakama found that recurrence was detected in $14.7 \%$ of spontaneous visits compared with $2.9 \%$ of regular visits conducted according to the recommended follow up schedule. They concluded that a woman is 5.1 times more likely to detect a recurrence herself than to have one detected during organised follow up. ${ }^{25}$ In this situation, educating patients about the indicators of relapse might be beneficial. Persistent pain and bone tenderness are common indicators of treatment failure, and patients should be taught to report such findings to their physician.

The possible detection of a contralateral primary is also regarded as an important component of follow up. The incidence of contralateral primaries is approximately $0.8 \%$ per year, ${ }^{21}$ and the survival of those with a localised contralateral

Table 3 Recurrence rate in each six month interval

\begin{tabular}{llllll}
\hline Interval (days) & $\begin{array}{l}\text { No of patients } \\
\text { entering } \\
\text { interval }\end{array}$ & $\begin{array}{l}\text { No developing } \\
\text { recurrence } \\
\text { during interval }\end{array}$ & $\begin{array}{l}\text { No ending } \\
\text { follow up during } \\
\text { interval }\end{array}$ & $\begin{array}{l}\text { No continuing } \\
\text { in follow up } \\
\text { beyond interval }\end{array}$ & $\begin{array}{l}\text { No at risk of } \\
\text { recurrence in } \\
\text { interval }\end{array}$ \\
\hline $1-180$ & 220 & 1 & 44 & 175 & 197.5 \\
$181-360$ & 175 & 7 & 14 & 154 & 164.5 \\
$361-540$ & 154 & 6 & 8 & 140 & 147.0 \\
in interval (\%)
\end{tabular}

*Since extraction of information on follow up was truncated at five years, it was not known how many patients had further follow up after five years. Thus, the number at risk and the recurrence rate in the last 180 day interval could not be estimated. 


\begin{tabular}{ll|}
\hline $\begin{array}{l}\text { Table } 4 \\
\text { Indicator }\end{array}$ & First indicators of recurrence \\
\hline Symptoms or finding of abnormality on self & $31(74)$ \\
examination & $2(5)$ \\
Follow up examination & $5(12)$ \\
Routine mammogram & $4(10)$ \\
Unknown or not in file & \\
\hline
\end{tabular}

primary is as good as that of those with a localised index tumour. ${ }^{26}$ In this study four patients developed contralateral tumours, which may have been new primary cancers or metastases from the previous primaries. All four tumours, however, were diagnosed during unscheduled visits. This is similar to the series reported by Loong et al and to the results of other studies, which found that routine clinic visits did not contribute directly to detecting contralateral tumours. ${ }^{16}{ }^{1721}$

While there was no very consistent pattern, the highest recurrence rates in this study were in the second and third years after diagnosis. Although this finding is supported by others, ${ }^{17}{ }^{19}$ it may be a result of selecting women who had five years follow up recorded in their notes.

This study did not assess the survival benefit of follow up. Tomin and Donegan and Dewar and Kerr retrospectively compared the survival of patients who developed asymptomatic recurrence with that of those who had symptomatic recurrence after mastectomy for early breast cancer..2021 Both groups reported better overall survival for patients with asymptomatic recurrences. However, this was attributed to lead time bias and length bias. More importantly, Tomin and Donegan's paper showed that there was no difference in survival between patients who complied with the schedule of follow up examinations and those who deviated from the schedule. These findings are in accordance with those of Boekhorst et al and others. ${ }^{15} 1922$ Thus, current evidence suggests that early detection and treatment of asymptomatic locoregional recurrence has no benefit to overall survival compared with treatment of symptomatic recurrence.

The need for psychosocial support for women with breast cancer is increasingly recognised. ${ }^{27}$ Psychological morbidity, particularly anxiety, is common, occurring in up to $30 \%$ of patients within a year of diagnosis. ${ }^{19}{ }^{28}$ In busy clinics these problems may be under-recognised. Psychological morbidity can be overlooked if it is not deliberately addressed. This support may be better and more consistently provided by general practitioners than by regular visits to an outpatient clinic. ${ }^{29}$ There is evidence that, although some patients do gain reassurance from clinic visits, a reduced frequency of follow up is preferred and that patients may be willing to accept follow up in primary care, particularly if provision is made for prompt specialist review should the need arise. $^{10} 29$

A randomised controlled trial by Grunfeld et al found that follow up care provided by general practitioners was as
Table 6 Referral pattern for unscheduled visits

\begin{tabular}{ll}
\hline Referral type & No (\%) of patients $(\mathbf{n}=\mathbf{3 2})$ \\
\hline Self referred & $17(53)$ \\
Referred by general practitioner & $10(31)$ \\
Referred by another specialist & $0(0)$ \\
Referred after routine mammography & $5(16)$ \\
\hline There were six scheduled visits, and information was not available for \\
four patients.
\end{tabular}

effective as that delivered by hospital based specialists in terms of quality of life and time to detection of distant metastases. They found that women detect most recurrences as interval events and present to the general practitioner, irrespective of continuing hospital follow up. ${ }^{29}$ However, there were only 26 events in 296 patients over the 18 month follow up, so the statistical power is limited. A survey forming part of this study showed that 69\% of general practitioners in England consider that they have the skills necessary to conduct routine follow up. A similar survey of Australian general practitioners would be helpful.

There is no evidence as to what constitutes optimal follow up; alternatives to hospital outpatient clinics have been proposed to address the various requirements of follow up, ${ }^{16} 17^{19}$ including monitoring treatment related toxicity, diagnosing and managing psychological problems, and collecting long term data. In countries such as Australia with a strong primary care base, one alternative is to give responsibility for routine follow up to the general practitioner (especially in rural areas), with the option to reinitiate specialist hospital care if problems develop. General practitioners in the UK have expressed a willingness to undertake this responsibility. ${ }^{29}$ However, this option may require further general practitioner education to improve follow up in primary care.

The current incidence of breast cancer is high. Early detection of primary cancers and the strict use of adjuvant therapy not only result in longer survival but also lengthen the disease-free interval, thus increasing the number of patients requiring follow up.

In this retrospective analysis and review of current evidence, a regular follow up schedule did not appear to offer an obvious advantage in the detection of recurrence after potentially curative treatment. Adequate quantification of late toxicity effects and of the value of psychological support cannot be ascertained from this study.

Any new approach to follow up must fulfil various requirements, including monitoring treatment related toxicity, diagnosing and managing psychological problems, and collecting long term data. From international research it appears that given adequate preparation both patients and general practitioners may be happy with early discharge, and this will not compromise surveillance for recurrence.

To change the follow up protocol for the management of primary breast cancer in Australia the following questions need to be considered:

\begin{tabular}{llllll} 
Table 5 & \multicolumn{2}{l}{ Recurrence by visit type and presence or absence of symptoms } \\
\hline & \multicolumn{2}{l}{ Scheduled visits } & & \multicolumn{2}{l}{ Unscheduled visits } \\
\cline { 2 - 3 } Site of recurrence & Symptomatic & Asymptomatic & & Symptomatic & Asymptomatic \\
\hline Locoregional & 2 & 1 & 3 & 3 \\
Metastatic & 2 & 1 & 21 & 1 \\
Contralateral breast & 0 & 0 & 3 & 1 \\
\hline \multicolumn{4}{l}{ Information was not available for four patients. }
\end{tabular}


- Can breast cancer follow up be adequately provided in the community?

- Are there any special categories of women who may benefit from hospital based follow up?

- What does evidence indicate should be the components of long term follow up, if any?

- What are the effects on long term outcomes such as mortality and morbidity of follow up by a specialist compared with follow up in primary care in Australia?

- Will Australian women accept follow up by the general practitioner?

- What are Australian general practitioners' views on follow up of cancer patients?

- What are the psychological effects of follow up?

\section{REFERENCES}

1 National Health and Medical Research Council. Clinical practice guidelines for management of early breast cancer. Canberra: NHMRC, 2001:97-102.

2 Wong WW, Vijayakumar S, Weichselbaum RR. Prognostic indicators in nodenegative early stage breast cancer. Am J Med 1992;92:539-48

3 Gasparini G, Bevilacqua P, Dal Fior S, et al. Characterization of nodenegative breast cancer by clinico-pathologic and biologic factors. Anticancer Res 1990;10:205-8.

4 Boyages J, Bosch C, Langlands A, et al. Breast conservation: longterm Australian data. Int J Radiat Oncol Biol Phys 1992;24:253-60.

5 Burke MF, Allison R, Tripcony L. Conservation therapy of breast cancer in Queensland. Int J Radiat Oncol Biol Phys 1995;31:295-303.

6 Pendlebury SC, Ivanov O, Renwick S, et al. Long-term review of a breast conservation series and patterns of care over 18 years. Aust N Z J Surg 2003;73:577-83

7 American Society of Clinical Oncology. Update of recommended breast cancer surveillance guidelines. J Clin Oncol 1999;17:1080-2.

8 Rojas MP, Telaro E, Russo A, et al. Follow-up strategies for women treated for early breast cancer (Cochrane review). In: Cochrane Library, Issue 3. Oxford: Update Software, 2002

9 The GIVIO Investigators. Impact of follow-up testing on survival and healthrelated quality of life in breast cancer patients. A multicentre randomized controlled trial. JAMA 1994;271:1587-92.

10 Rosselli Del Turco M, Palli D, Cariddi A, et al. Intensive diagnostic follow-up after treatment of primary breast cancer. A randomized trial. National
Research Council project on breast cancer follow-up. JAMA 1994;271:1593-7.

11 Gulliford T, Opomo M, Wilson E, et al. Popularity of less frequent follow-up for breast cancer in randomised study: initial findings from the hotline study. BMJ 1997:314:171-7.

12 Schapira DV. Breast cancer surveillance-a cost-effective strategy. Breast Cancer Res Treat 1993;25:107-11

13 Muss HB, McNamara MJC, Connelly RA. Follow-up after stage II breast cancer: a comparative study of relapsed versus non-relapsed patients. Am J Clin Oncol 1988;11:451-5.

14 Rosner D, Lane WW. Predicting recurrence in axillary-node negative breast cancer patients. Breast Cancer Res Treatt 1993;25:127-39.

15 te Boekhorst DS, Peer NG, Van der Sluis RF, et al. Periodic follow-up after breast cancer and the effect on survival. Eur J Surg 2001;167:490-6.

16 Loong S, Wilkins M, Bliss JM, et al. The effectiveness of the routine clinic visit in the follow-up of breast cancer patients: analysis of a defined patient cohort. Clin Oncol 1998;10:103-6.

17 Donnelly J, Mack P, Donaldson LA. Follow up of breast cancer: time for a new approach? Int J Clin Pract 2001;55:431-3.

18 Pandya KJ, McFadden ET, Kalish LA, et al. A retrospective study of earliest indicators of recurrence in patients on Eastern Cooperative Oncology Group adjuvant chemotherapy trials for breast cancer. A preliminary report. Cancer 1995;55:202-5.

19 Churn M, Kelly V. Outpatient follow-up after treatment for early breast cancer: updated results after 5 years. Clin Oncol 2001;13:187-94.

20 Tomin R, Donegan WL. Screening for recurrent breast cancer-its effectiveness and prognostic value. J Clin Oncol 1987;5:62-7.

21 Dewar A, Kerr GR. Value of routine follow-up of women treated for early carcinoma of the breast. BMJ 1985;291:1464-7.

22 Clemons M, Danson S, Hamilton T, et al. Locoregionally recurrent breast cancer: incidence, risk factors and survival. Cancer Treat Rev 2001; 27:67-82.

23 Fisher B, Baver M, Margolese R, et al. Five-year results of a RCT comparing total mastectomy and segmental mastectomy with or without radiation in the treatment of breast cancer. N Engl J Med 1985;312:665-8.

24 Morris S, Corder AP, Taylor I. What are the benefits of routine breast cancer follow-up? Postgrad Med J 1992;68:904-7.

25 Holli K, Hakama M. Effectiveness of routine and spontaneous follow-up visits for breast cancer. Eur J Cancer Clin Oncol 1989;25:251-7.

26 Robinson E, Rennert G, Rennert HS. Survival of first and second primary breast cancer. Cancer 1993;71:172-6.

27 National Health and Medical Research Council. Psychosocial clinical practice guidelines. Canberra: NHMRC, 2000.

28 Kiebert GM, Welvaart K, Kievit J. Psychological effects of routine follow-up on cancer patients after surgery. Eur J Surg 1993;159:601-7.

29 Grunfeld E, Mant D, Yudkin P, et al. Routine follow up of breast cancer in primary care: randomised trial. BMJ 1996;313:665-9. 